\title{
Recomendaciones para el manejo de las malformaciones arteriovenosas cerebrales
}

\author{
Actualización y modificaciones a las recomendaciones del Comité Vascular de la Fede- \\ ración Latinoamericana de Neurocirugía (FLANC) del año 2003
}

\author{
E. Spagnuolo; L. Lemme-Plaghos*; F. Revilla**; L. Quintana*** y J. Antico****
}

Neurocirugía vascular. Uruguay. *Neurocirugía endovascular. Argentina. **Neurocirugía vascular México. ***Neurocirugía vascular. Chile. ****Neurocirugía. Radiocirugía. Argentina.

\section{Resumen}

Las malformaciones arteriovenosas son un grupo de lesiones bastante heterogéneo, que representan un desafío para el neurocirujano.

La variabilidad de su tamaño, topografía, angioarquitectura, etc., así como las diferentes posibilidades de presentación clínica, hacen que los planteos terapéuticos sean variados.

No hay consenso a nivel internacional incluso de cómo tratar malformaciones de características similares. Influye también en esto las diferentes opciones terapéuticas que se ofrecen y la experiencia que se tiene en el tratamiento de las mismas.

Es por todo lo anterior, que la Federación Latinoamericana de Neurocirugía, a través de su Comité Vascular en el año 2003 publicó unas primeras pautas de manejo de las malformaciones arteriovenosas.

Los avances en el diagnóstico y en el tratamiento que se han verificado en los últimos años, motivó a los autores a revisar esas primeras pautas y actualizarlas.

Hay que tener en cuenta que lo que se presenta son solamente guías para que un neurocirujano tenga algo en que apoyarse al enfrentarse ante una lesión de este tipo, pero de ninguna manera se pretende que las mismas deban cumplirse estrictamente.

PALABRAS CLAVE: Malformaciones arteriovenosas cerebrales. Guías de tratamiento.

Recomendation for the management of the brain arteriovenous malformations

Summary

Intracranial arteriovenous malformations constitute an heterogenous group of lesions that represent a real challenge to the neurosurgeon.

Their variability in size, topography, angioarchitec-

Recibido: 17-04-08. Aceptado: 15-07-08 ture, etc., and also the multiple clinical presentations, lead to varied therapeutic approaches.

There is no international consent regarding how to treat malformations of similar features. This fact is influenced by the different therapeutic options, and the personal experience in the management of these lesions.

Based on the former considerations, the FLANC (Latin American Federation of Neurosurgery), through its Vascular Commitee, published in 1993 the first guidelines in the management of arteriovenous malformations.

The advances in the diagnosis and treatment verified in the past last years, has motivated the authors to review and actualize these first guidelines.

The intention of these guidelines is to provide the neurosurgeon support when facing these lesions, and does not mean that they should be strictly followed.

KEY WORDS: Intracranial arterioveous malformations. Guidelines of treatment.

Introducción

En el año 2003, el Comité Vascular de la Federación Latinoamericana de Neurocirugía, presidido por el Dr. Leónidas Quintana publicó las primeras recomendaciones para el manejo de las malformaciones arteriovenosas cerebrales. Para estas recomendaciones se realizó un exhaustivo estudio de la bibliografía publicada al respecto, a lo que se sumó la experiencia personal de quienes realizaron el trabajo ${ }^{28}$.

Las pautas fueron ideadas para servir como base para el desarrollo de las estrategias de tratamiento de las malformaciones arteriovenosas (MAV).

Las recomendaciones son exclusivamente para las MAV cerebrales y piales ${ }^{28}$. No se aplican a otro tipo de malformaciones vasculares cerebrales y craneanas como los angiomas transicionales y sindromáticos, los angiomas cavernosos, angiomas venosos (anomalías de drenaje venoso), malformaciones arteriovenosas y fístulas durales, 
ni las fístulas arteriovenosas traumáticas (ej. Fístulas carótido cavernosas) que presentan etiología, clínica e historia natural diversa.

\section{Pautas de manejo}

Las MAV representan desde el punto de vista angio arquitectónico a un grupo heterogeneo de lesiones cerebrovasculares, que se pueden manifestar clínicamente de diferentes maneras ${ }^{41}$.

El continuo avance que se da en la terapéutica endovascular, así como la opción de la terapia radiante mediante radiocirugía y los resultados a largo plazo de las mencionadas, sumado a los resultados quirúrgicos, han servido para que los autores propongan algunas modificaciones a las actuales "pautas".

Lo que si es seguro, es que con el arsenal terapéutico con que se cuenta, salvo el caso de algunas malformaciones, para la mayoría hay tratamiento y con alto porcentaje de curación.

Las malformaciones arteriovenosas son lesiones probablemente de origen o predisposición congénita caracterizadas por un conglomerado de vasos anormales (arterias y venas), de tamaño y número variables caracterizados por la ausencia de red capilar normal intermedia. Entre los vasos anormales no hay parénquima y la periferia de la lesión está rodeada de un tejido gliótico ${ }^{11,19,35,42}$. El conocer la existencia de este tejido modificado, alrededor de una malformación es muy importante, ya que da un margen de seguridad para el tratamiento quirúrgico cuando el mismo se realiza, independientemente de la topografía de la lesión. En el caso de las MAV superficiales existe asimismo un espesamiento de la aracnoides suprayacente ${ }^{14}$.

Otro elemento importante a tener en cuenta cuando uno se enfrenta a una MAV, es el concepto de que estas no son lesiones "estáticas", sino que tienden a aumentar de volumen con el tiempo por un reclutamiento de estructuras vasculares próximas, a expensas de los vasos, tanto arteriales como venosos que forman parte de la malformación o que se vinculan a las mismas. Las MAV sufren aumento de calibre por fenómenos de hiperflujo y alteraciones en sus paredes, que las hacen más frágiles, con tendencia a la formación de seudo o verdaderos aneurismas intranidales o en las arterias aferentes. Estas modificaciones favorecen las complicaciones en estas lesiones, con claro predominio de las hemorragias ${ }^{2,3,10,17}$.

En los diferentes trabajos publicados no hay unanimidad respecto a la incidencia y prevalencia de las MAV, pero se puede concluir que entre el $1,4 \%$ y el $4,3 \%$ de la población sería portadora de esta patología. También es variable el porcentaje de las malformaciones sintomáticas ${ }^{1,4,23,26}$.

Hay que tener en cuenta que según la bibliografía internacional en el mundo occidental se dan por año unos 200 accidentes vasculares encefálicos cada 100.000 habitantes, de los cuales el 20\% son hemorrágicos. Dentro de estos últimos el $75 \%$ corresponden a hematomas cerebrales vinculados a hipertensión arterial, el 15\% corresponden a hemorragias subaracnoideas y aproximadamente el $6 \%$ son por sangrados de malformaciones arteriovenosas. El $4 \%$ restante se reparte entre otras afecciones que se pueden complicar con sangrados cerebrales. Resumiendo, si el 6\% serían sangrados por malformaciones arteriovenosas, equivaldría a que hay 2 casos de hemorragias por rotura de MAV cada 100.000 habitantes y por año ${ }^{1,21}$.

Hay que destacar que si bien la hemorragia es la forma más común de presentación clínica de una MAV, y también la más grave, éstas se pueden presentar con otros síntomas. Los más comunes (además de la ya mencionada hemorragia) $\operatorname{son}^{31,40,42}$ :

- cefaleas

- epilepsia

- isquemia cerebral por robo

- trastornos neurosíquicos

- soplos

- insuficiencia cardíaca (en la edad pediátrica)

- hidrocefalia no comunicante (por venas intraventriculares dilatadas por hiperflujo)

- "Incidentales" (estas últimas, si bien no corresponde a forma clínica sintomática de presentación, es una manera de diagnosticarse una MAV cuando se hace un estudio, por ejemplo TC o RM por otra causa y se encuentra la malformación).

Para el manejo de las MAV se sigue utilizando como guía la escala de Spetzler y Martin, publicada en $1986^{32}$. Si bien se han propuesto algunas modificaciones y existen algunas críticas a la misma, sigue siendo un referente mundial para todo el que se enfrenta a una MAV. Quizás el punto más controvertido de esta clasificación son las malformaciones que los autores incluyen dentro del grado III. Hay algunas de este grado que son corticales y de tratamiento relativamente sencillo, pero también hay grado III que son profundas, pequeñas en áreas elocuentes o del tronco cerebral, en donde el tratamiento y las opciones terapéuticas son discutibles ${ }^{3,14,20,24}$. La escala de Spetzler se refiere fundamentalmente a lesiones con expresión cortical e insular.

Por ese motivo es que en los años posteriores a la publicación de la mencionada escala se han propuesto modificaciones en este grado. Es así que hay quienes las dividen en IIIa a las corticales y IIIb a las profundas u otros que colocan estas últimas en otro grado (VI) ${ }^{20,24,37}$.

Como es bien conocido, la clásica clasificación (escala) de Spetzler y Martin, pondera tres elementos: el tamaño de la malformación, la localización y el tipo de drenaje venoso.

De esta manera se llega a 5 grados de MAV: 


\section{Escala de Spetzler-Martin}

\section{Tamaño}

$0-3 \mathrm{~cm} \ldots \ldots \ldots \ldots \ldots . . .1$

$3,1-6 \mathrm{~cm} \ldots \ldots \ldots \ldots . .2$

$+6 \mathrm{~cm} \ldots \ldots \ldots \ldots \ldots . . . . .3$

\section{Localización}

Área no elocuente ...0

Área elocuente .......1

\section{Drenaje venoso profundo}

No presente ...........

Presente ..............1

En otras palabras, se puede decir que las malformaciones grado I y II son pequeñas, o no tan pequeñas, pero de resección quirúrgica relativamente fácil y en las que no se debería discutir su tratamiento por esta técnica en manos

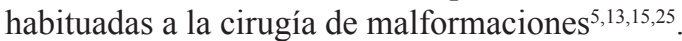

En el otro extremo las malformaciones grado IV y V son las grandes y las "gigantes" y ante las cuales se discute si se debe encarar un tratamiento activo o no. Es en estas malformaciones donde se deben considerar otras variables técnicas para plantear tratamientos o no, como se expondrá mas adelante ${ }^{12,30,33}$.

Por su parte las MAV grado III se deben tratar, pero son las que generan más discusión, ya que su variabilidad en tamaño, topografía y drenaje, hace que los planteos terapéuticos sean disímiles ${ }^{24,30,31,34}$.

Con respecto a cómo efectuar las recomendaciones, el grupo que formuló las pautas de manejo, tomó en cuenta en la extensa bibliografía consultada y desde el punto de vista estadístico, los niveles de evidencia y la fuerza de los datos acumulados ${ }^{28}$ :

\section{Niveles de evidencia}

Nivel 1: datos de estudios randomizados con bajos errores falso positivos (alfa) y bajos errores falso negativos (beta)

Nivel 2: datos de estudios randomizados con altos errores falso positivos y altos errores falso negativos

Nivel 3: datos de estudios de cohorte concurrentes no randomizados

Nivel 4: datos de estudios de cohorte utilizando controles históricos no randomizados

Nivel 5: datos de series de casos anecdóticos

\section{Estudios diagnósticos}

Las MAV se pueden diagnosticar mediante diferentes estudios imagenológicos.

\section{Fuerza de datos acumulados}

Grado A: sostenido por el nivel de evidencia I

Grado B: sostenido por el nivel de evidencia II

Grado $C$ : sostenido por el nivel de evidencia III, IV y V

Una vez hecho el diagnóstico, es necesario completar el estudio de la MAV mediante otros procedimientos imagenológicos, para poder tener una clara noción de las características de la lesión: su angioarquitectura, su anatomía, topografía exacta y las relaciones con las estructuras vecinas.

\section{Tomografia computada:}

Una tomografía computada (TC) sin contraste, es un estudio de sensibilidad baja en el diagnóstico de estas lesiones. Puede hacer sospechar la lesión al verse imágenes patológicas, como ser calcificaciones, imágenes espontáneamente hiperdensas redondeadas, serpinginosos o la existencia de un área de secuela parenquimatosa, con zonas de mayor densidad en su seno. Con la administración de contraste se puede hacer diagnóstico, pero no aclara sobre detalles de la lesión. Con los equipos actuales de tomografía ("multislice") se pueden hacer reconstrucciones tridimensionales del cerebro y su vasculatura que ponen en evidencia la malformación, la presencia o no de aneurismas intranidales, determinar detalles de irrigación y de angioarquitectura de la lesión. La ventaja de este método, es que es un estudio mínimamente invasivo, ya que requiere exclusivamente de la inyección intravenosa de contraste. Puede ser suficiente como estudio diagnóstico para malformaciones pequeñas, pero no para aquellas grado III o más, para las que se necesitan estudios dinámicos como la angiografía digital, para planificar un tratamiento adecuado ${ }^{3,14}$.

\section{Resonancia magnética}

Es un procedimiento diagnóstico muy sensible para estas lesiones. Las reconstrucciones en varios planos y en las diferentes ponderaciones permiten una correcta visualización de la lesión, su exacta topografía y su angioarquitectura. Permite además poner en evidencia la existencia de lesiones parenquimatosas adyacentes a la malformación, como áreas de gliosis reaccional o la presencia de lesiones isquémicas perimalformación. Por su parte la angio-resonancia puede brindar detalles sobre la irrigación de la malformación, mediante un método diagnóstico no invasivo $^{14,18}$.

\section{Angiografía digital de vasos del cuello y cerebro}

Es el estudio principal para estas lesiones. Da detalles de su anatomía, de sus arterias aferentes y de las venas de drenaje. La angiografía supraselectiva muestra detalles 
hemodinámicos y fisiológicos importantes para el análisis clínico y para la toma de decisiones. Además mediante este procedimiento diagnóstico se puede planificar una eventual terapia endovascular. Es en definitiva el estudio diagnóstico mas importante. Siempre debe incluirse la exploración angiográfica de las carótidas externas, ya que muchas veces suministran aferencias durales a las malformaciones cuando estas tienen expresión cortical ${ }^{22,25,29}$.

En resumen se recomienda para el diagnóstico de una malformación arteriovenosa:

1.- Angiotomografía con reconstrucción 3D.

2.- Resonancia Magnética (con o sin angioresonancia).

3.- Angiografía digital selectiva de vasos de cuello y encefálicos, con estudio de carótida externa bilateral.

\section{Clínica e historia natural}

Como ya fue expresado es variada la forma de presentarse clínicamente una MAV, sobresaliendo ampliamente el debut con una hemorrágia. Esta forma de presentación clínica se da en aproximadamente el 50\% de los casos. Predomina el sangrado parenquimatoso, aunque también pueden verse hemorragias subaracnoideas o intraventriculares exclusivamente.

La epilepsia se presenta de inicio entre un 20\% y un $25 \%$ de los casos, en un $15 \%$ se presentan con cefaleas, mientras que el 5\% debuta con un déficit focal neurológico ${ }^{3,4,10,42}$.

Con respecto al riesgo anual de sangrar una malformación diagnosticada, pero que no sangró varía según las diferentes estadísticas entre un $2 \%$ y un $4 \%$. Estos porcentajes se consideran acumulativos anualmente, por lo que cuanto más joven es el paciente, y por ende con mayor expectativa de vida, se impone el tratar la malformación. También es diferente el caso de aquella malformación que se diagnostica porque se volvió sintomática, pero sin sangrar, que aquéllas en donde el diagnóstico es casual, al estudiar al paciente por otra razón, como por ejemplo el realizar una TC de cráneo por un traumatismo. Las malformaciones sintomáticas tienen más riesgo de sangrar que aquellas que son asintomáticas ${ }^{4,9}$.

Cuando se está en presencia de una malformación que sangró y no se trató, el riesgo de una nueva hemorragia es muy superior a lo expresado precedentemente, variando el riesgo, según diferentes autores entre un $6 \%$ y un $17 \%$ en el primer año luego del primer sangrado ${ }^{4,42}$.

Ante un caso determinado además del riesgo anual de sangrado se debe considerar el riesgo de sangrado por vida restante en base al siguiente cálculo:

riesgo durante la vida $(\%)=105$ menos edad del paciente ${ }^{10}$.

Hay elementos clínicos e imagenológicos que se consideran predictores de un eventual sangrado.

Desde el punto de vista clínico se mencionan la apari- ción de signos focales neurológicos, o la agravación de los ya existentes.

Desde el punto de vista imagenológico, los más consistentes son la existencia de malformaciones pequeñas, la presencia de nidos periventriculares o intraventriculares, la constatación de un drenaje venoso profundo o único $\mathrm{y}$ la presencia de aneurismas o seudoaneurismas arteriales o venosos, sobre todo los intranidales $2,4,13,15,17,26,40$.

\section{Riesgos de tratamiento versus beneficios}

Múltiples factores deben ser tenidos en cuenta en el momento de decidir si tratar o no una malformación, así como cúal es el método o la combinación de ellos que puede ser el indicado en cada caso en particular.

Lo primero a tener en cuenta y como se dijo más arriba es la historia natural de la enfermedad, la edad del paciente, la existencia de elementos predictores de sangrado y los porcentajes de morbimortalidad que esta eventualidad tiene. Por otro lado en caso de decidirse por tratarlas, hay que tener en cuenta los resultados que se tienen con cada una de las opciones terapéuticas que se ofrecen. Para esto último no se pueden establecer pautas generales, ya que incide en forma significativa la experiencia y la disponibilidad de medios que se tiene en cada Centro Asistencial en las diferentes opciones que se ofrecen.

En el caso de tomarse la decisión de realizar un tratamiento, hay que tener en cuenta que éste debe buscar la resección u oclusión completa de la lesión, y que los porcentajes de riesgo que se manejen con el tratamiento deben ser menores a los riesgos naturales de la enferme$\operatorname{dad}^{5,25,34,36,41}$.

Por lo tanto, cuando uno se enfrenta a una MAV se pueden plantear diferentes opciones:

- Conducta expectante

- Tratamiento médico sintomático (ej. anticomiciales)

- Resección quirúrgica

- Terapia endovascular

- Radiocirugía Estereotáxica

- Combinación de procedimientos

Se desprende de lo anterior, que para llevar adelante una terapia adecuada, se debe contar con un equipo multidisciplinario que discuta cada caso en particular y las diferentes opciones, de manera de brindar al paciente las mayores garantías posibles.

Desde otro punto de vista se puede dividir el tratamiento en curativo y en paliativo.

Un tratamiento curativo es aquél que asegura la eliminación completa de la malformación y es al que debe aspirarse cuando un equipo se decide a tratar una $\mathrm{MAV}^{5,7,13,25}$. Pero existen lesiones en que por su tamaño, características, 
topografía, etc., la cura es imposible y en esos casos los tratamientos son parciales, paliativos. Lo que se busca con éstos es disminuir el riesgo de complicaciones. El caso más típico dentro de esta última opción es una malformación arteriovenosa grado $\mathrm{V}$, topografiada fundamentalmente a nivel profundo y con múltiples sangrados que ponen en riesgo la vida del paciente. En un caso como este, la terapia endovascular, con oclusión de parte del ovillo, puede determinar para algunos autores una disminución del riesgo de sangrado $^{6,12}$. Esto no es aceptado por otros, que plantean que posterior a la embolización, al ocluir vasos anormales de mayor calibre, se transformaría una MAV de alto flujo y baja resistencia en una de alta resistencia, con el consiguiente aumento de riesgo de sangrado ${ }^{16,18}$.

$$
\begin{array}{r}
\text { Tratamiento de una MAV } \ldots . . . \ldots \ldots \text { Curativo } \\
\ldots \ldots \ldots \ldots \ldots \text { Paliativo }
\end{array}
$$

Para hablar de curación se debe aceptar que se ha eliminado la malformación y esto se logra con cirugía. Desde el punto de vista angiográfico y con terapia endovascular, en algunos casos podemos hablar de "oclusión completa angiográfica", pero esto no es sinónimo de desaparición de todo el ovillo, ya que pequeños vasos aferentes pueden quedar presentes, y no verse en el estudio imagenológico, lo que hace suponer la "curación", pero a partir de esos pequeños vasos se puede reproducir en tiempo variable la lesión $^{6,37,38,39}$.

Hay equipos que pueden optar por terapia endovascular, seguida de radiocirugía. Esta combinación puede llevar a la desaparición de la lesión, pero hay que saber que para que se llegue a esto, hay que esperar hasta 2 años luego de la terapia radiante, lo que determina que en ese período de tiempo puede haber sangrados. Riesgo este, como ya fue expresado, mayor si la MAV tratada había sangrado ${ }^{8}$.

En manos experimentadass, la cirugía de una MAV es una intervención sistematizada, donde se debe previamente estar "familiarizado" con la lesión, con sus vasos aferentes y eferentes. Se debe hacer siempre la resección siguiendo el cono que habitualmente forma el ovillo y manteniéndose siempre por fuera del mismo, para evitar sangrados del nido. Se debe ir "eliminando" progresivamente los vasos arteriales y siempre dejar para el final la vena principal de drenaje. En las MAV grandes no es infrecuente perder el plano e irse en la resección hacia adentro del ovillo, esto se manifiesta en la cirugía por sangrados importantes. Ante esta situación no se debe insistir con la coagulación, sino que hay que cubrir la zona e ir por fuera en búsqueda del plano entre la lesión y el cerebro. Por lo general de esta manera se domina el sangrado y se puede seguir en forma reglada la resección de la malformación ${ }^{30,31,42}$.

De acuerdo a lo que se expone en las pautas y en lo que los autores están totalmente de acuerdo, es que el trata- miento de una MAV es un tratamiento de coordinación ${ }^{28}$.

No es recomendable realizar una cirugía de urgencia de una MAV.

Uno se puede enfrentar a una malformación en una situación de emergencia, cuando sangró y hay un hematoma que sí debe ser evacuado. En ocasiones en que es una emergencia incluso puede no contarse con otro estudio más que una TC, pero si se logra realizar una angio TC o incluso una angiografía antes de la cirugía del hematoma, se debe planificar el acto quirúrgico, de manera de evacuar el hematoma en un punto lo más alejado posible del ovillo. Hay casos en que son MAV muy pequeñas y se pueden extirpar conjuntamente con el hematoma pero esto se da en contadas ocasiones. De todas maneras el concepto es que en la urgencia se debe evacuar el hematoma y se debe dejar la cirugía de la MAV para la coordinación, una vez que el paciente esté recuperado de la hemorragia y de la cirugía a la que fue sometido ${ }^{28}$.

Con respecto a la cirugía de una MAV, independientemente del grado de la misma, siempre se deben seguir las técnicas micro quirúrgicas estándares.

Como lo que se busca es la resección completa, que asegure la curación, es altamente recomendable contar con angiografía intra operatoria, ya que la constatación de un pequeño remanente obliga a ir en su búsqueda para resecarlo. En caso de no contarse con angiografía intra operatoria, se debe realizar este estudio en el postoperatorio, lo más precoz posible, para actuar en consecuencia en caso de remanentes. A este respecto la conducta a seguir, cirugía complementaria o radiocirugía, dependerá de la topografía del resto de MAV, la condición del paciente y la angioarquitectura de ese remanente. Estos por lo general son pequeños, profundos y quedan en cirugías de MAV grandes ${ }^{16}$. Es en estos casos en que se recomienda la complementación con radiocirugía estereotáxica.

En aquellos Servicios en los cuales se cuente con la posibilidad de efectuar un tratamiento endovascular pre operatorio el mismo es aconsejable en los casos de MAV grado II, III y IV porque la disminución obtenida por embolización del volumen de la MAV aunque mas no fuese parcial facilita el acto quirúrgico por disminución de la turgencia de las venas de drenaje y facilitación de la disección del nido angiomatoso ocluido, lo que disminuye el sangrado intraoperatorio acortando los tiempos quirúrgicos.

Con respecto a los resultados quirúrgicos, la mayoría de los trabajos, así como la experiencia personal (ES), está basada en recopilaciones de datos de nivel $\mathrm{V}$ (estudios retrospectivos $)^{30}$. Sin embargo, y en base a la escala de Spetzler y Martin, se puede estimar el riesgo con la cirugía dependiendo del grado de la lesión.

Los trabajos publicados, y con clara orientación quirúrgica, estiman resultados favorables con los siguientes porcentajes dependiendo del grado: 
- MAV grado I: $92 \%$ a $100 \%$

- MAV grado II: 95\%

- MAV grado III: $68 \%$ (a corto plazo) y $88,6 \%$ (a largo plazo)

- MAV grado IV: 73\% a largo plazo

- MAV grado V: 57\% a largo plazo

- MAV grado IIIb o VI: no hay datos claros, salvo series pequeñas, donde con la ayuda de guía estereotáxica hacen referencia a MAV ganglio basales, etc. Pero las de tronco cerebral, por lo general no llegan a la cirugía y en caso de tratarse, se lo hace con terapia endovascular y/o radiocirugía ${ }^{20,34}$.

Con respecto a la opción de radiocirugía, la misma tiene sus indicaciones, en algunos casos precisos, mientras que en otros es discutida. De todas maneras, se pueden establecer algunas pautas para la indicación de la radiocirugía:

1. Está indicada en MAV de volumen inferior a $8 \mathrm{cc}$ y si lo consideramos mas estrictamente a MAV con una integral de dosis (es decir la relación dosis / volumen, que se expresa en Milijoules) de 170mJ. Esto sería comparable al volumen de $8 \mathrm{cc}$.

2. Que la MAV este localizada en el tronco cerebral, y que ocupe un volumen no mayor del 30\% del total del tronco.

3. Que esté situada a una distancia de protección con respecto a estructuras críticas como el aparato óptico.

4. Es de limitada indicación en caso de hemorragias y no aconsejable si tuvo más de una hemorragia en menos de 6 meses, siempre y cuando existan alternativas terapéuticas (cirugía y / o tratamiento endovascular).

5. No está contraindicada en casos de hallazgo asintomático en donde no es necesario reducir con embolizaciones, salvo que sea mayor de $8 \mathrm{cc}$.

6. Cuando sólo existiera un comienzo clínico con epilepsia, comparte primera indicación con otras modalidades (cirugía - embolización).

7. Está indicada en pacientes que presentan patologías concomitantes que impidan una cirugía cuando estuviera indicada.

Basándonos tanto en la experiencia personal, como en los trabajos de más reciente publicación, la cirugía en las MAV grado $\mathrm{V}$ no está indicada ${ }^{12,31}$. La excepción está dada en aquellas lesiones córtico subcorticales con hemorragias frecuentes, y que las mismas pongan en riesgo la vida del paciente o que ya hayan determinado un déficit neurológico severo. En caso contrario estas MAV no se deberían tratar, o a lo sumo serían de indicación de terapia paliativa. Por lo tanto una MAV grado $\mathrm{V}$, sin mayores complicaciones en principio no se trata y son objeto de observación, de vigilancia clínica e imagenológica ${ }^{31}$.

Cuando hay que realizar tratamientos, los mismos están encabezados por la terapia endovascular. Como ya fue expresado es un tratamiento paliativo, que busca reducir el flujo en una MAV muy grande, reducir su tamaño, eliminar en lo posible aneurismas asociados (intranidales o no). Se puede complementar este tratamiento con radiocirugía del sector no embolizado. Varios trabajos se han publicado con la combinación de estas dos técnicas, pero también han aparecido otros en que se demuestran malos resultados,

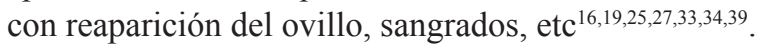

En resumen: si es una lesión sintomática, con o sin sangrado, pero que pone en riesgo la vida del paciente se opta por terapias alternativas, paliativas (neurocirugía endovascular y radiocirugía). La cirugía directa de estas malformaciones en principio se debe evitar. Su realización se limita a casos excepcionales, como ya fue expresado (múltiples sangrados, secuelas severas). Tener en cuenta que la cirugía de las MAV grado $\mathrm{V}$, siempre debe ser precedida de varias sesiones de terapia endovascular buscando la reducción del volumen del nido angiomatoso propiamente dicho, tal cual fue mencionado previamente. Siempre se debe aclarar al paciente y a la familia que es una cirugía difícil, con riesgos altos y con porcentajes altos de secuelas.

Las MAV grado IV, en general si son lesiones córtico subcorticales, que han sangrado se deben tratar. Por supuesto que este tratamiento siempre es combinado (terapia endovascular, cirugía y eventualmente radiocirugía). Tienen alto riesgo de secuelas neurológicas con la cirugía $^{12,30}$.

En el caso de MAV grado IV sintomáticas, pero sin sangrado, también se pueden tratar con las opciones antes mencionadas. Pero aquí entra a pesar la topografía de la lesión: área elocuente, ovillo profundo o no, topografía y relación de las venas de drenaje, etc.

Aquellas MAV grado IV que no han sangrado y que no son sintomáticas, o presentan síntomas menores (por ejemplo cefaleas), la observación clínica es la indicación. No consideramos los tratamientos paliativos en estos casos.

La neurocirugía endovascular sola o combinada con radiocirugía, no da garantías de curación y tienen sus riesgos, tanto en el procedimiento terapéutico como en la evolución posterior al tratamiento, ya que no lograrán la curación y la eventualidad de recrecimiento y sangrado estará siempre presente $6,8,19,34$.

Como ya fue expresado, las malformaciones arteriovenosas grado III son el grupo de lesiones que más controversias provocan en los grupos interdisciplinarios que las pueden tratar.

Son lesiones que se curan con el tratamiento, pero hay que tener en cuenta que no se pueden establecer pautas generales con respecto a todas las MAV grado III, ya que este grupo y como fue dicho, incluye un variado tipo de lesiones, ya sean superficiales como profundas, con drenaje 
venoso variable, etc (grados IIIa y IIIb o VI).

De todas maneras hay que saber que la enorme mayoría de las mismas se curan tratándolas con combinación de terapias (neurocirugía endovascular, radiocirugía estereotaxica, cirugía) y con un bajo porcentaje de riesgo de secuelas $^{34}$.

Son lesiones en las que la cirugía directa debe ser precedida por neurocirugía endovascular. No se deberían intervenir quirúrgicamente sin la mencionada terapia previa a la cirugía.

Siempre se logra con una o dos sesiones de terapia endovascular una importante reducción del flujo, tanto de las aferencias arteriales y del drenaje venoso, lo que cambia totalmente la cirugía. A pesar de lo anterior, hay MAV grado III que se han operado y se seguirán interviniendo directamente sin procedimientos endovasculares ${ }^{36,38}$. Todos los neurocirujanos con dedicación a neurocirugía vascular, saben que pueden llevar adelante una cirugía de una MAV grado III (sobre todo si es cortical o subcortical) sin terapia endovascular y con buenos resultados. Pero también es bien sabido por todos el beneficio obtenido con la neurocirugía endovascular ("embolización super selectiva") y que indiscutiblemente hay un antes y un después en el tratamiento de las MAV con el advenimiento de la terapia endovascular ${ }^{38}$. Los permanentes avances técnicos en la materia, como es el perfeccionamiento en micro catéteres, la innovación en materiales para embolizar y la mayor experiencia de los neurocirujanos endovasculares, llevan a la conclusión de que la terapia endovascular es una opción válida y muchas veces imprescindible el tratamiento de estas lesiones.

Como se mencionara previamente, la embolización super selectiva tiene la ventaja de que disminuye el sangrado intraoperatorio y que disminuye el tiempo quirúrgico. Todo esto porque ocluye vasos que pueden ser difíciles de dominar durante la cirugía. Disminuye lentamente la irrigación de la malformación, permitiendo la progresiva redistribución de flujo sanguíneo cerebral, ocluye aneurismas intranidales y ocluye eventuales fístulas de alto flujo $^{37}$.

Obviamente los tratamientos endovasculares también tienen riesgo de complicaciones, o sea que hay desventajas que a veces llevan a que se prescinda de la terapia endovascular. Dentro de estas desventajas cabe consignar: las hemorragias intra o post procedimiento; complicaciones isquémicas secundarias a oclusión de vasos gruesos, trombosis arterial o venosa y migración de los materiales de embolización a senos durales y a pulmones ${ }^{6,38,39}$.

Las malformaciones arteriovenosas grado II, son lesiones por lo general de ovillo pequeño. En caso de no tener un ovillo pequeño, su topografía no ofrecería a priori mayores riesgos de secuelas y su drenaje venoso debería ser superficial, siguiendo lo que marcan los diferentes puntos de la escala de Spetzler-Martin. Es por esto que este grado de malformaciones arteriovenosas, son pasibles de tratamiento curativo con cirugía. La misma se puede llevar adelante en forma directa sin terapia endovascular previa. De todas maneras recomendamos la neurocirugía endovascular preoperatorio en las MAV grado II (a excepción de aquéllas que tengan pedículos muy difíciles de cateterizar). Una malformación tratada previamente es sinónimo de una cirugía mucho más sencilla.

No se recomienda la terapia endovascular como única opción, ni tampoco la misma seguida de radiocirugía, ya que como fue mencionado previamente, la primera puede asegurar una oclusión completa angiográfica, pero esto no es sinónimo de oclusión real de la lesión. Complementar la mencionada oclusión angiográfica con radiocirugía, sí puede llevar a la curación, pero la acción radiante que permitirá esto, puede llevar hasta 2 años. Si el tratamiento fue realizado en una MAV que sangró, durante ese período sabemos que puede darse un nuevo sangrado, que incluso puede matar al enfermo. Todo esto en un grado de MAV donde sabemos que la cirugía tiene un porcentaje de curación superior al 95\% y con una morbimortalidad despreciable, por lo que no es planteable el tratamiento de una MAV grado II que excluya la cirugía directa ${ }^{28,30}$.

Con respecto a las MAV grado I, sucede algo similar a lo mencionado precedentemente con las grado II. Son lesiones eminentemente quirúrgicas. Se curan con cirugía y los riesgos de secuelas o de muerte son extremadamente bajos, se podría agregar que en manos experimentadas son despreciables otras opciones.

Por lo anterior, el plantear tratar con neurocirugía endovascular estas lesiones no tiene sentido. Por definición, por lo que es su tamaño, drenaje y localización, no ofrecen mayores dificultades a un neurocirujano. Sí se puede decir que dado que muchas veces son lesiones muy pequeñas, se puede asociar la localización estereotáxica, lo que facilita enormemente la cirugía.

Respecto a no operarlas e ir directamente a la radiocirugía, es bien sabido que esta opción terapéutica a la larga cura estas lesiones, pero como fue expresado en más de una oportunidad, si el diagnóstico se realizó por un sangrado, es muy peligroso decidirse por esta opción, por el tiempo que pasa hasta que se logra el cierre total de la misma. Por otro lado, si el diagnóstico no fue por sangrado, se podría ir directamente a la radiocirugía ${ }^{8}$, pero siempre teniendo en cuenta, que si bien el porcentaje anual de sangrado de una MAV es bajo, hasta que no pasen 2 años de tratada, esa lesión puede sangrar. En definitiva, en MAV grado I, la cirugía directa es la indicación.

De más esta decir, que estas pautas que se presentan, son siempre basadas en resultados de neurocirujanos, terapistas endovasculares y radiocirujanos que tienen una amplia experiencia en el manejo de las MAV y con un número alto de casos tratados. Indefectiblemente si el que se enfrenta a 
una MAV no tiene la suficiente experiencia y una amplia formación en la técnica que propone, se debe optar por otra de las opciones terapéuticas, aunque no sea la primera que aparece en las pautas. Como todo en neurocirugía, el conocimiento de la patología, la formación y el número de casos tratados son fundamentales en el momento de decidir cual es el mejor tratamiento a realizar. No hay que dudar en referir a un paciente con una MAV a un colega que tenga la suficiente experiencia en alguna de las posibilidades terapéuticas mencionadas. De otra manera, los resultados nunca serán los esperados.

En resumen:

\section{Diagnóstico:}

1) TC "multislice" con estudio vascular (reconstrucciones tridimensionales

2) RM con angioresonancia

3) Angiografía de vasos de cuello y cerebro (incluye carótidas externas)

Opciones terapéuticas:

* No hacer nada (control clínico e imagenológico). Tratamiento expectante

* Tratamiento médico de los síntomas

* Cirugía

* Neurocirugía endovascular

* Radiocirugía estereotáxica

* Combinación de alguna de las antes mencionadas.

Tratamiento según el grado:

\section{MAV grado $I$.}

1) Cirugía directa (primera opción)

2) Radiocirugía (si no sangró: segunda opción)

3) Neurocirugía endovascular (tercera opción). Puede lograr la "curación angiográfica", pero no asegura la oclusión real de la malformación.

4) Tratamiento expectante: control clínico e imagenológico, no debería plantearse. Solo aceptable si las condiciones del "medio" donde se encuentra el paciente no ofrece las opciones precedentes.

Porcentaje de curación cercano al 100\%.

\section{MAV grado II}

1) Cirugía directa (primera opción)

2) Neurocirugía endovascular, opción valida como eventual terapia preoperatoria o prerradiocirugía. Nunca como única opción.

3) Radiocirugía. Nunca como primera o única opción, puede hacerse luego de terapia endovascular. Las limitaciones están en los riesgos del sangrado durante el "tiempo de acción” del tratamiento.

4) Tratamiento expectante. Con las mismas condicio- nes y limitaciones de lo mencionado para las grado I. Porcentaje de curación cercano al 100\%.

\section{MAV grado III.}

1) Tratamiento combinado: Neurocirugía endovascular y cirugía. (Primera opción)

2) Cirugía directa (sin procedimientos endovasculares previos), se puede realizar, pero con porcentaje alto de secuelas. No es recomendable.

3) Neurocirugía endovascular exclusiva, no es recomendable. No logra la curación. Siempre va a volver a formarse el ovillo y los riesgos a corto plazo serán los mismos que sin tratamiento; pero el paciente corrió riesgos de secuelas con el procedimiento.

4) Neurocirugía endovascular como parte del tratamiento, es lo indicado. Principalmente como paso previo a la cirugía. Eventualmente como paso previo a la radiocirugía.

5) Radiocirugía. En principio no es primera opción. Puede serlo en aquellas pequeñas malformaciones profundas que no sangraron o que habiendo sangrado el riesgo quirúrgico sea sumamente alto. Un ejemplo de esto es una pequeña malformación arteriovenosa del tronco cerebral (IIIb o VI). La otra opción de radiocirugía es cuando el paciente no accede a la cirugía luego de la terapia endovascular.

Porcentaje de curación cercano al 100\%, pero tienen hasta un $25 \%$ de secuelas definitivas.

MAV grado IV.

1) Si no sangró y no hay elementos predictores de sangrado, se recomienda la observación (control clínico-imagenológico).

2) Si sangró: siempre tratarlas.

Tratamiento combinado: (endovascular + cirugía + radiocirugía) es la opción terapéutica.

$1^{\circ}$ - Neurocirugía endovascular (siempre más de una sesión).

$2^{\circ}$ - Cirugía (siempre y cuando sea una malformación accesible.

$3^{\circ}$ - Radiocirugía (siempre después de la neurocirugía endovascular). En este caso estamos hablando de tratamiento paliativo. Radiocirugía luego de terapia endovascular y cirugía, en este caso será curativa, ya que se indicará para un pequeño remanente.

Cabe consignar que: la neurocirugía endovascular exclusiva, es una terapia paliativa y en principio no indicada en este grado. La cirugía directa, es una opción poco recomendable. Son cirugías muy sangrantes y con un muy elevado porcentaje de secuelas

Con el tratamiento combinado: porcentaje de curación 
cercano al 100\%, pero con morbilidad de más del 45\%.

\section{$M A V$ grado $V$.}

En principio no se tratan. Los riesgos del tratamiento son mayores que los riesgos naturales de la enfermedad.

Tratamiento: siempre es combinado. Se plantea exclusivamente en aquellas malformaciones que presentan sangrados reiterados, que están poniendo en riesgo la vida del paciente. La cirugía se incluye dentro de este tratamiento combinado solamente en aquellas lesiones córtico subcorticales. En los casos en que se hace tratamiento combinado con endovascular, cirugía y radiocirugía se puede lograr la curación, pero el $100 \%$ de los casos presentará secuelas neurológicas. Se destaca que cuando se llega a tratar estos enfermos, casi siempre ya presentan lesiones neurológicas definitivas, secundarias a sangrados e isquemia.

Mayoritariamente cuando hay que hacer algo, es un tratamiento paliativo e incluye: neurocirugía endovascular (varias sesiones) y radiocirugía.

\section{Bibliografía}

1. Bastarrica, E.: Epidemiología de los Accidentes Vasculares Hemorrágicos Encefálicos. En: Curso Sobre Accidentes Cerebro-Vasculares Hemorrágicos. Ed. Sandoz, Montevideo 1989; 3-18.

2. Batjer, H., Suss, R., Samson, D.: Intracranial AVM's associated with aneurysms. Neurosurgery 1986; 18: 29-35.

3. Brown, R., Flemming, K., Meyer, F. et al.: Natural History, Evaluation and management of Intracranial Vascular Malformations. Mayo Clin Proc 2005; 80: 269-281.

4. Crawford, P., West, C., Chadwick, C., et al.: Arteriovenous malformations of the brain: natural history in unoperated patients. J. Neurol. Neurosurg Psychiatry 1986; 49: 1-10.

5. Cromwell, L., Harris, A.: Treatment of cerebral arteriovenous malformations:. A combined neurosurgical and neuroradiological approach. J. Neurosurg 1980; 52: 705-708.

6. Deveikis, J.: Endovascular therapy of intracranial arteriovenous malformations. Material and methods. Neuroimaging Clin N Am 1998; 8: 401-424.

7. Dion, J., Mathis, J.: Cranial arteriovenous malformations: the role of embolization and stereotactic surgery. Neurosurg Clin N Am 1994; 5: 459-474.

8. Flickinger, J., Pollock, B., Kondziolka, D., et al.: A dose related analysis of arteriovenous malformation obliteration after radiosurgery. Int J Radiat Oncol Biol Phys 1996; 873-879.

9. Fox, A., Viñuela, F.: Neurological deficit from inoperable arteriovenous malformation: an indication for therapeutic embolization? Arch. Neurol 1986; 43: 510-511.

10. Graf, C., Perret, G., Torner, J.: Bleeding from cerebral arteriovenous malformations as part of their natural history J.Neurosurg 1983; 58: 331-337.
11. Gualt, J., Sarin, H., Shenkar, R., Awad, I.: Pathobiology of human cerebrovascular malformations. Neurosurgery 2004; 55: 1-16.

12. Han, P.P., Ponce, F., Spetzler, R.: Intention -to- treat analysis of Spetzler-Martin grades IV and V arteriovenous malformations: natural history and treatment paradigm. J. Neurosurg 2003; 98: 3-7.

13. Hartmann, A., Mast, H., Mohr, J.: Morbidity of intracranial hemorrhage in patients with cerebral AVM's. Stroke 1998; 29: 931-934.

14. Hashimoto, N., Nosaki, K., Takagi, Y.: Surgery of Cerebral AVM's. Neurosurgery 2007; 61: 375-389.

15. Heros, R., Korosue, K., Diebold, P.: Surgical excision of cerebral AVM's. Late results. Neurosurgery 1990; 26: 570578 .

16. Jafar, J., Davis, A.A., Berenstein, A. et al.: The effect of embolization with N-butyl cyanoacry prior to surgical resection of cerebral arteriovenous malformations. J. Neurosurg 1993; 78: 60-69.

17. Kader, A., Young, W., Pile Spellman, J., et al.: The influence of hemodynamis and anatomic factors on hemorrhage from cerebral arteriovenous malformations. Neurosurg 1994; 34: 801-807.

18. Kucharczyk, W., Lemme-Pleghos, L., Uske, A.: Intracranial vascular malformations- MR and CT imaging. Radiology 1985; 56: 383-389.

19. Lanman, T., Martin, N., Vinters, H., et al.: The pathology of encephalic arteriovenous malformation treated by prior embolotherapy J Neuroradiol 1988; 30: 1-10.

20. Lawton, M., Hamilton, M., Spetzler, R.: Multimodality treatment of deep AVM's: Thalamus, basal ganglia, and brain stem. Neurosurgery 1995; 37: 29-35.

21. Mast, H., Young, W., Koennecke, H.: Risk of spontaneous hemorrhage after diagnosis of cerebral AVM's. Lancet 1997; 350: 1065-1068.

22. McCormick, WF., Schochet, S.: Atlas of cerebrovascular disease. Philadelphia. Pa: WB Saunders Co 1976:422.

23. Michelson, Wj.: Natural history and pathophysiology of AVM's. Clin. Neurosurgery 1978; 26: 307-313.

24. Nosaki, K., Hashimoto, N., Kikuta, K.: Surgical application to arteriovenous malformations involving the brainstem. Neurosurgery 2006; 58: 270-279.

25. Ogilvy, C., Stieg, P., Awad, I., Brown, R.: Recommendation for the management of Intracranial AVM's. A Statement for Healthcare Professionals from a special Writing group of the Stroke Council, American Stroke Association. Stroke 2001; 32: 1458-1471.

26. Ondra, SL., Troupp, H., George, E.: The natural history of symptomatic AVM's of the brain. J.Neurosurg 1990; 73: 387-391.

27. Pollock, B., Flickinger, J.: A proposed radiosurgery based grading system for arteriovenous malformations. J. Neurosurg 2002; 96: 79-85. 
28. Quintana, L., Revilla, F., Pedroza, A., Antelo, G., Giocoli, H.: Recomendaciones para el manejo de las Malformaciones Arteriovenosas 2003. Pag.Web Flanc. www.eflanc.com.

29. Russell, D., Rubinstein, L.: Pathology of tumors of the Nervous System 3 ${ }^{\text {ra }}$. Edición. Pags. 93-102 William \& Wilkins, Baltimore, 1971.

30. Spagnuolo, E., Calvo, A., Lin, T.: Malformaciones Arteriovenosas Encefálicas. Tratamiento definitivo SIIC Salud. www.siicsalud.com. 2001.

31. Spagnuolo, E.: Malformaciones Arteriovenosas. En: Spagnuolo, E.-Calvo,A.: Patología Vascular Hemorrágica Encefálica Ed: Of. Del Libro. Montevideo. 1997; 115-138

32. Spetzler, R., Martin, N.: A proposed grading system for AVM J.Neurosurg. 1986; 65: 476-483.

\section{Comentario al trabajo Recomendaciones para el manejo de las malformaciones arteriovenosas cerebrales de $\mathbf{E}$. Spagnuolo y cols.}

A veces, no es conveniente seguir las recomendaciones "al pie de la letra" porque el plan terapéutico de las MAV es uno de los aspectos más difíciles y subjetivos de la Neurocirugía.

Recientemente, Spetzler ha simplificado la escala publicada en 1986 junto con Martin en tres tipos: A, que corresponde a los antiguos grados I y II, donde el tratamiento de elección es la cirugía; B, que corresponde al grado III, que es un grupo de MAV muy heterogeneo, algunas con clara indicación de tratamiento quirúrgico y otras que se pueden tratar con embolización y/o radiocirugía; $\mathrm{C}$, que son las MAV grado IV y V que en muchos casos la mejor opción es la abstención terapéutica.

Después de esta simplificación cada especialista puede individualizar el tratamiento, es decir, puede abstenerse en un anciano con una MAV grado I y operar una MAV grado IV superficial que sangró.

Las cifras de incidencia que refieren los autores nos parecen un tanto exageradas. Los patrocinadores del estudio ARUBA han publicado tasas de detección de 1.34 por 100.000 habitantes/año.

Corresponde a Robert D. Brown el mérito de la formula
33. Spetzler, R., Martin, N., Carter, L., et al.: Surgical management of large AVMs by staged embolization and operative escision. J. Neurosurg 1987; 67: 17-28.

34. Steiner, L., Lindquist, C., Cail, W., et al.: Microsurgery and Radiosurgery in brain arteriovenous malformations. J. Neurosurg 1993; 79: 647-652.

Spagnuolo, E.; Lemme-Plaghos, L.; Revilla, F.; Quintana, L.; Antico, J.: Recomendaciones para el manejo de las malformaciones arteriovenosas cerebrales. Neurocirugía 2009; 20: 5-14.

Correspondencia postal: Dr. Edgardo Spagnuolo. Jefe del Servicio de Neurocirugía. Hospital Maciel, 25 de mayo s/n. CP.: 11000 Montevideo, Uruguay.

: 105 - edad del paciente para saber el riesgo durante la vida de un paciente determinado, aceptando el riesgo del 2-4\% anual (publicado en Neurosurgery, abril 2000).

La embolización preoperatoria es muy debatible, de acuerdo con Heros no debe abusarse para que el neurocirujano tenga "un buen dia" y el paciente sume los riesgos de los dos procedimientos cuando con sólo la cirugía puede alcanzarse el mismo objetivo. No obstante, en este punto cada equipo debe saber cuantos enfermos fallecen por una embolización parcial cuando el plan de tratamiento era embolización + cirugía.

Por último, creo que no se debería hacer ningún plan de tratamiento de ninguna MAV sin el acuerdo de un neurocirujano experto/dedicado a patología neurovascular. En otras palabras, no se puede embolizar indefinidamente una MAV sin el objetivo de curarla ni tratar con radiocirugía una MAV de $3.5 \mathrm{~cm}$. cortical en un paciente joven sin proponerle antes un tratamiento quirúrgico.

J. Vilalta Barcelona 